AperTO - Archivio Istituzionale Open Access dell'Università di Torino

\title{
Knowledge-Based Framework for the Virtual Communication of Public Art
}

\section{This is the author's manuscript}

Original Citation:

\section{Availability:}

This version is available http://hdl.handle.net/2318/1577791

since 2016-11-30T10:53:32Z

Publisher:

Institute of Electrical and Electronics Engineers Inc.

Published version:

DOI:10.1109/IMIS.2015.22

Terms of use:

Open Access

Anyone can freely access the full text of works made available as "Open Access". Works made available under a Creative Commons license can be used according to the terms and conditions of said license. Use of all other works requires consent of the right holder (author or publisher) if not exempted from copyright protection by the applicable law. 
This is the author's final version of the contribution published as:

Lombardo, Vincenzo; Damiano, Rossana; Lieto, Antonio. Knowledge-Based Framework for the Virtual Communication of Public Art, in: Proceedings 2015 9th International Conference on Innovative Mobile and Internet Services in Ubiquitous Computing, IMIS 2015, Institute of Electrical and Electronics Engineers Inc., 2015, 9781479988730, pp: 117-124.

The publisher's version is available at:

http://xplorestaging.ieee.org/ielx7/7282217/7284886/07284936.pdf?arnumber=7284936

When citing, please refer to the published version.

Link to this full text:

http://hdl.handle.net/2318/1577791 


\title{
Knowledge-based framework for the virtual communication of public art
}

\author{
Vincenzo Lombardo, Rossana Damiano, and Antonio Lieto \\ CIRMA and Dipartimento di Informatica \\ Università di Torino, Torino, Italy \\ Email: vincenzo.lombardo@unito.it, rossana@di.unito.it, lieto@di.unito.it
}

\begin{abstract}
This paper deals with the documentation and communication of contemporary public art. It analyzes the specific requirements of such a form of cultural heritage and presents a system that implements the visualization of implicit information in a web-based virtual reality environment. The core of the system is a knowledge-based (ontological) module, devised to represent the values, the realization procedures, and the access ways to public art items. The visualization system relies upon the ontological representation to provide a 3D layout that takes into account the relevant traits that characterize the contemporary public art. The issues of integration of the public art within the urban context is the object of a visualization component, implemented in interactive web-based 3D graphics, that relies on the ontological knowledge concerning the public art, integrated with additional ontological descriptions of spatial geographic knowledge.
\end{abstract}

\section{INTRODUCTION}

Public art is an exciting area of investigation for virtual reality representations because of its tight relationship with the natural or urban environments. In the last decades, public art has been a very dynamic area, contrasting with the tendency to host art in museums, involving both public and private subjects and originating a novel professional artist category. Public art has the peculiarity of being planned and staged in some public place and specific space, usually open air or in publicly accessible buildings and it is accessible to all people [1]. In this paper, we propose a virtual environment as the pivotal interface to address documentation and communication of contemporary public art. The virtual environment relies on a semantic knowledge base that integrates information about the artworks, necessary to the documentation task, and geo-spatial information, necessary to generate the visual equivalent of the artwork, displayed within its urban context. The joint use of these sources of information, made interoperable by the use of semantic web techniques, yields a user experience that joins information and fruition in an integrated virtual environment.

This is not the prime use of computer graphics for contemporary public art. The computer graphics representation is ubiquitously employed in the design phase, for the previsualization of projects (see, e.g., Figure 1): the major issues of the project are selected to point out the features of the artwork, such as the size of the artwork, the materials employed, how it looks from a number of notable points of view, the ways of access, etc. . Virtual environments are also particularly appropriate for the case of reconstructions of cultural heritage items from the past, such as in the case of [2], that has regained the original audiovisual experience of the Poème électronique, by Le Corbusier, Varèse, and Xenakis,

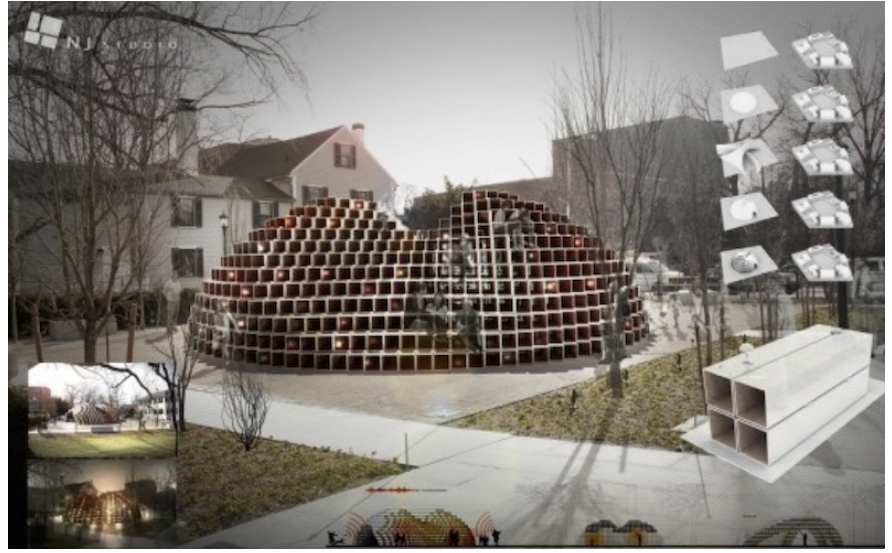

Fig. 1. reStacked: manufactured landscape from recycled materials. Project presented by the NJstudio, in collaboration with Aurgho Jyoti, Matan Mayer, Daekwon Park, for the Public art competition (2013), the Radcliffe Institute for Advanced Study at Harvard University, MA, USA. Courtesy of NJstudio.

a multimedia installation that was hosted inside the Philips Pavilion at the 1958 Brussels World Fair, and never repeated because the pavilion was turned down at the end of the fair. The virtual reconstruction employs 3D real time graphics and spatialized audio to deliver a sense of the original space.

Cataloguing activities, developed since the XIX century by cultural institutions and national entities[3], imply underlying conceptual models can be expressed in an explicit way using formal representation tools [4]. With the advent of the digital era and the complexity and multidisciplinarity of contemporary installations, the need for an explicit representation has moved from the mere description of the artworks to the definition of processes and actors for purposes of documentation of the cultural heritage. The ontology languages designed as part of the Semantic Web initiative [5] has described conceptual models in an unambiguous way, open to understanding and manipulation of human users and software programs [6]. A number of research projects have explored the application of semantic technologies to cultural heritage, starting with the pioneering initiative of the Finnish Culture Sampo project [7]. Conceived as a large-scale demonstrator, Culture Sampo is a cultural heritage portal entirely relying on a "mash up" of domain ontologies that encode all the relevant features of artworks, from geographical data to craftsmanship. In the case of public art, the documentation of cultural heritage needs to be augmented with information about the spatial context of the artwork and the possible functional properties 
In this paper, we present a framework for the communication of public art, that joins the requirements of documentation and communication. The documentation about the public art is encoded in a computational ontology, that is interpreted as a virtual scene that works as an environment for the communication and promotion of the public art items. The paper is organized as follows. The next section introduces the computational ontologies for the public art, extending a widely acknowledged model in the literature on the documentation of cultural heritage. Then, we address the issue of providing a virtual environment for the communication of the public art, that results from an interpretation of the knowledge encoded in the extended ontology. Finally, we provide some application example and conclusions.

\section{COMPUTATIONAL ONTOLOGIES FOR PUBLIC ART}

In order to work out a conceptual model for some area of the cultural heritage, one needs to start from the widely acknowledged CIDOC CRM model. Being compliant with CIDOC CRM allows the conceptual model to inherit a large number of features that are shared by all forms of cultural heritage and to operate in the wide context of a reference framework. In addition, this design choice makes the extended model built upon CIDOC CRM interoperable and therefore potentially re-usable for similar purposes. Issued by the International Council of Museums (ICOM), the CIDOC Conceptual Reference Model (CIDOC CRM) is intended as a "common language for domain experts and implementers", specifically aimed at the design of information systems [4]. Developed along more than two decades by the CIDOC Documentation Standards Working Group (DSWG), CIDOC CRM is currently released in a semantic format ${ }^{1}$ that supersedes the previous conceptual model encoded in the relational data model. In this section, we review the major issues of the model and then introduce the extensions required to represent the public art. In particular, we start from the notion of tangible cultural heritage, then we introduce the so-called intangible components, and finally we extend the ontology to the graphic model that represents the spatial context in which the item is embedded and to the geographic coordinates that locate the item in a public space.

The top level of CIDOC CRM includes five classes: TimeSpan, Place, Dimension, PersistentItem, and TemporalEntity. ManMadeThing (subclass of PersistentItem) encompasses the classes for representing PhysicalObjects and ConceptualObjects, further subdivided into the PropositionalObject and SymbolicObject classes. The TemporalEntity class includes the Event class, a subclass of which is the Activity class. The Actor class, encompassing the participants to events, is a subclass of the PersistentItem class. The documentation of CIDOC CRM illustrates the functioning of the model through an example: the bronze statue "Monument to Balzac" by Auguste Rodin (follow concepts and relations in Figure 2). The monument itself is represented as an Information Carrier (i.e., it is an instance of the Information Carrier class, subclass of the above mentioned ManMadeThing) and it depicts a Person, Honoré de Balzac. The statue has material bronze and was produced through a Production process (the bronze casting, a type of Activity, and, as such, a subtype of class Event), carried

\footnotetext{
${ }^{1}$ http://www.cidoc-crm.org/docs/cidoc_crm_version_6.0.pdf
}

out by a Legal Body (the company that made the bronze casting, name Rudier et Fils). The bronze casting continued a previous Production (the plaster modeling) carried out by a Person, Auguste Rodin himself. As this example illustrates, CIDOC CRM is not limited to the representation of the internal structure of the artwork, but it opens to the reference of real world entities that are not intrinsically related to the art domain, such as physical persons and historical events. It also represents in a fine-grained way the production processes that have originated the artwork and the roles played in them by various actors, including individuals and groups.

Shifting to contemporary art, CIDOC CRM must account for further issues that were not envisaged at the design time of the model. The first issue concerns the representation of the relations between a work (that in contemporary art can also be a performance, or an installation) and its documentation, which is the result of the process of documenting the art, a practice strongly encouraged by the current availability of digital media (and prevalently consisting of pictures/video captured during the design/production activity and during the exhibition/performance). The second issue that has emerged in contemporary art is the requirement of providing explicit instructions for the maintenance of the artwork, a step that is often necessary due to the more transitory nature of many contemporary artworks, which involve disposable components such as, e.g., light bulbs or neon tubes. Documentation and maintenance are highly relevant issues for the impermanent components that characterize contemporary art, cf. [8]. Contemporary art is characterized by the commixture of installations (often impermanent), performances and interactive elements. Specific inadequacies of CIDOC CRM emerged regarding the modeling needs of interactive installations and live performances. These inadequacies often refer to the notion of reproducibility, given that the artwork design can be reproduced several times, with variations brought in by some different context and participants. For example, the case study illustrated below, Merz's igloo shaped fountain in Turin instantiates one of the variations of the igloo shape, conceived by the artist.

Our proposal of extension relies on the introduction of new properties to describe some "invisible" components of contemporary artworks and on putting them in relation with the item. Previous extensions of the CIDOC CRM models can be found in the literature. For example, [8] proposed an extension aimed at modeling the notion of reliability and provenance in the transfers of possession of cultural heritage items (introducing the modeling of roles involved in such processes over time); [9] has introduced the notion of performance into the ontology and [10] suggests that the FRBR model is suitable to account for the problem of variation (in performances and installations):

"The problem of variation is the problem of how, if a Work is defined by all the examples of it, we can determine that two examples that are not identical are nonetheless part of the same Work. This problem is especially pronounced in live performance, which, by its very nature, has the potential for each of its examples to be unique."

In fact, aspects such as reproducibility and serial production of contemporary art are dealt with by the model known as "Functional Requirements for Bibliographical Records" (FRBR), described in [11]. Designed for capturing "the under- 


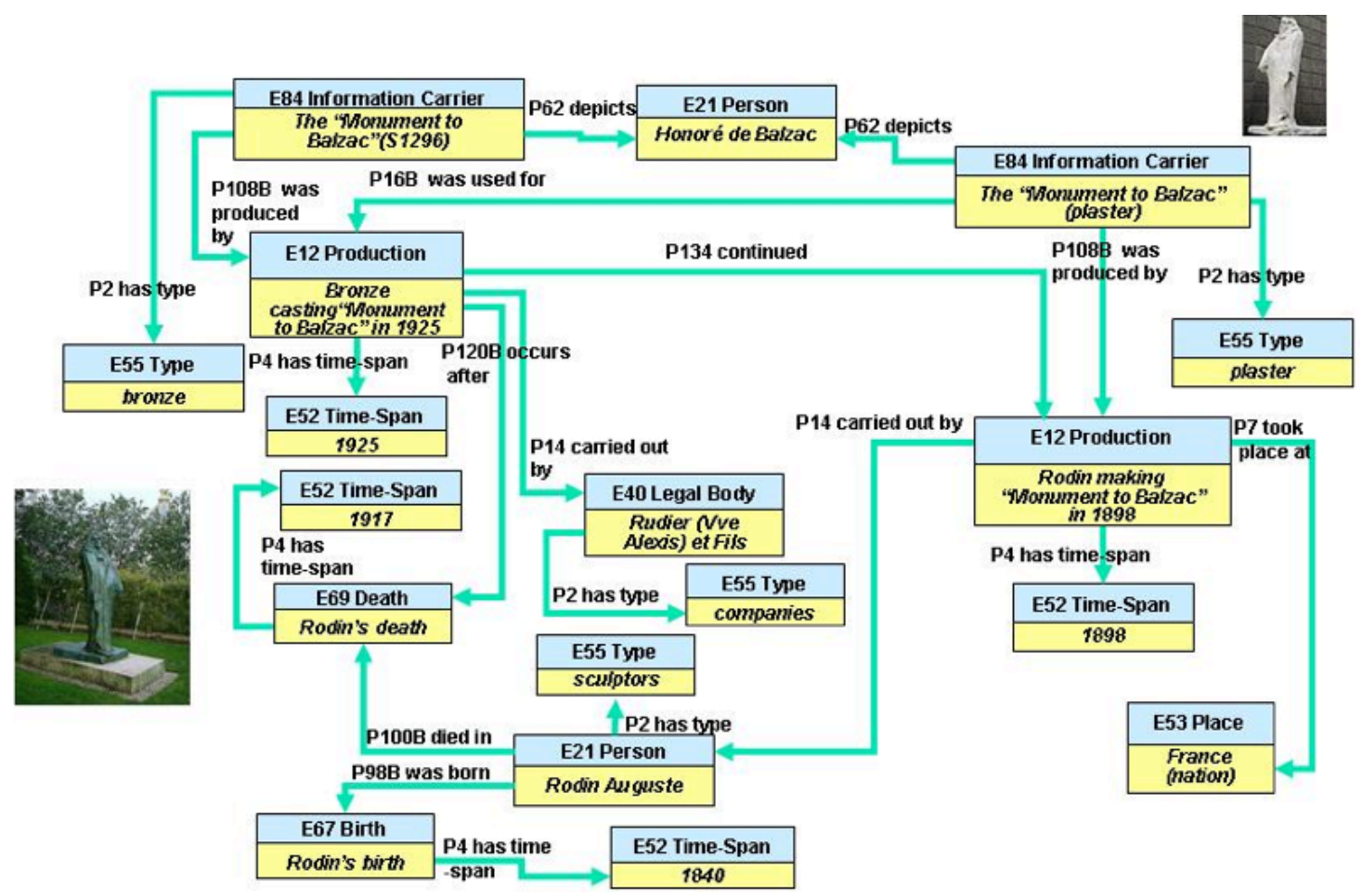

Fig. 2. Modeling Rodin's "monument to Balzac" in CIDOC CRM.

lying semantics of bibliographic information”, FRBR encompasses four main entities, mainly Work, or abstract ideation, Expression, that is the encoding of the Work in a specific language such as text or music, Manifestation, its embodiment in a concrete representation, and Item, a single manifestation in an editorial process. We have included the FRBR model in the ontology, relying on the mapping of the FRBR model onto the CIDOC CRM proposed by the FRBRoo working group[12]. According to this proposal, the notion of Work corresponds to the ConceptualObject class in CIDOC CRM, and different versions of the same work are represented as instances of the Manifestation class, which corresponds to the SymbolicObject class in CIDOC CRM.

The second issue concerns the encoding of a specific spatial (mostly urban) context for the contemporary public artworks, that is also relevant for the case of a visualization task in a virtual environment: on the one hand, geographic coordinates are needed to locate the artworks and the items that compose the visual scene in the visual interface; on the other, the 3D models and shapes (in general, extruded polygons) provide the context objects that have to be positioned in the virtual scene according to the geographical information. Geographic coordinates are related to some map that represents the background against which 3D models and item shapes are positioned for the presentation to the user.

In Figure 3, there is a sketch of these extensions of the ontology: the upper part of the sketch (with respect to the class "Artwork") is a reference to the CIDOC CRM FRBR model; the lower part is the extension that accounts for the visualization issues. Each artwork is associated with a corresponding visual Scene that is composed of several Items and is visually represented by a Model (a 3D model, in particular). Each model has geographic coordinates (imported from the Geoportal of the city, in the case of Public Art, on the left), size parameters, and an IRI (Internationalized Resource Identifier). The IRI is a reference to some address for the actual (3D) model, typically a Collada file.

The spatial structure of the visual scene is on the lower right of Figure 3. A Scene is composed of visual Items. Each item is either a distinct 3D Model or a Shape, that is a polygon, composed of vertices at some geographic coordinates. A shape can have parameters that define the visual aspect of the item: for example, the densities of the streetlights as well as of trees of an area can be set to some value ("low" or "high", respectively, in the figure) and set manually or interpreted procedurally by the visualization module. A shape can be an abstract infrastructure or a Geoportal infrastructure (i.e., externally described in some repository of geographical data). Paths and animations are abstract infrastructures: a path (realized, e.g., by a spline) provides a site along which things happen; an animation occurs along a temporized path (which is in turn a form of path) and features an actual model that moves along the path. Paths and animations are useful to represent visually the notion of access to the artwork. We introduced two types of paths and animations, respectively: the pedestrian access, that animates a stylized human model along a path, the car access, that animates a stylized vehicle along a path (see below) The Geoportal infrastructures are elements that can be extracted from some urban scale repository of geographical data, such as green areas, buildings, functional elements. Functional elements concern information that is useful for visualization; in fact, some elements of the spatial context do not receive a position from the Geoportal data, and we need to yield their positions through their function with respect to other elements. The ontology sketch in Figure 3 


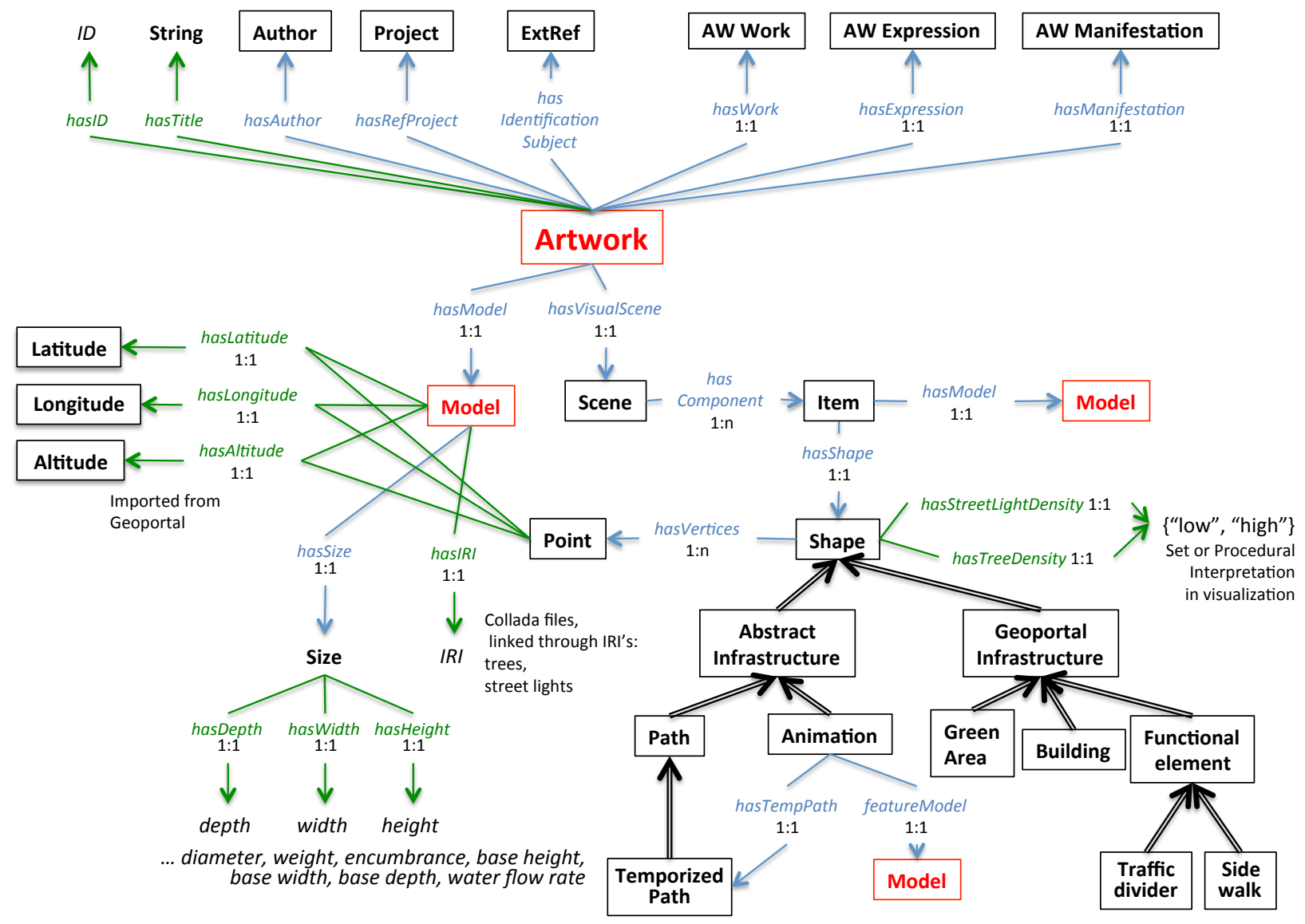

Fig. 3. Tripartite ontology of public art, oriented to virtual reality representation. In the upper part, the reference to CIDOC CRM - FRBR model (AW = Artwork); in the lower left part, the geographic section; in the lower right part, the spatial ontology, with the items that populate and animate the artwork context.
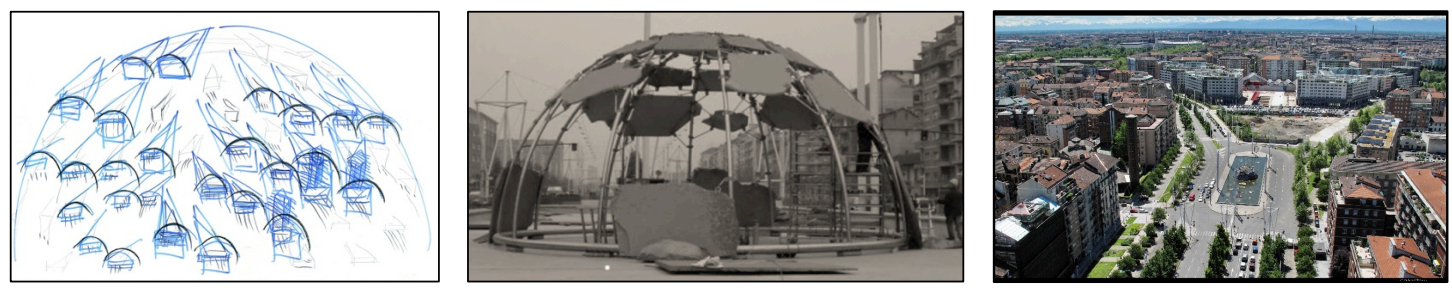

Fig. 4. Merz's igloo fountain in Turin: three paradigmatic images for Work (left), Expression (middle), Manifestation (right), respectively.

samples two types of functional elements, sidewalks and traffic dividers. Sidewalks stand aside of a road, while traffic dividers split a road into two carriages.

We have applied the extended model to four examples of public art during the project "Invisibilia" (see Acknowledgments). Here, we illustrate the artwork "Fontana" (Fountain) of the artist Mario Merz (see Figure 4). The Fountain of Mario Merz is a public artwork in Turin (Italy), released in 2002, that has the shape of an igloo with the surface consisting of a puzzle of plates of slate, emerging from a rectangular water tab, with water jets, located in a road widening; four red neon lights, that light up at evening, mark the cardinal points. In the ontology of Public Art, Metz's fountain is an instance of PropositionalObject/Work class, and is described by a ProceduralPrescription (executive specifications) to which it is connected via the property isRealizedIn. The manifestation of the idea (instance of the SymbolicObject/Manifestation class) is given by an installation made of plates of slate and neon tubes, whose size, layout, etc., also described in the ontology. The physical artwork, then, is related to its maintenance activity (instance of MantainanceActivity), documented by some specific document (instance of the MaintenanceDocumentation class), and distinct (for time, location, actors) from the CreationActivity that originated the monument, which has a different Time and Location. We have introduced the class Score with the goal of representing scripted, complex artistic performances. In the case of Merz's Fountain, this class is employed to describe the enlightening of neon lights in the evening. In geo-spatial terms, the igloo fountain of Mario Merz is a traffic divider, as reported by the catalogue card in the city hall archives. Also we know that the fountain is surrounded by green areas, sidewalks and buildings, specific types of Geoportal Infrastructure. 


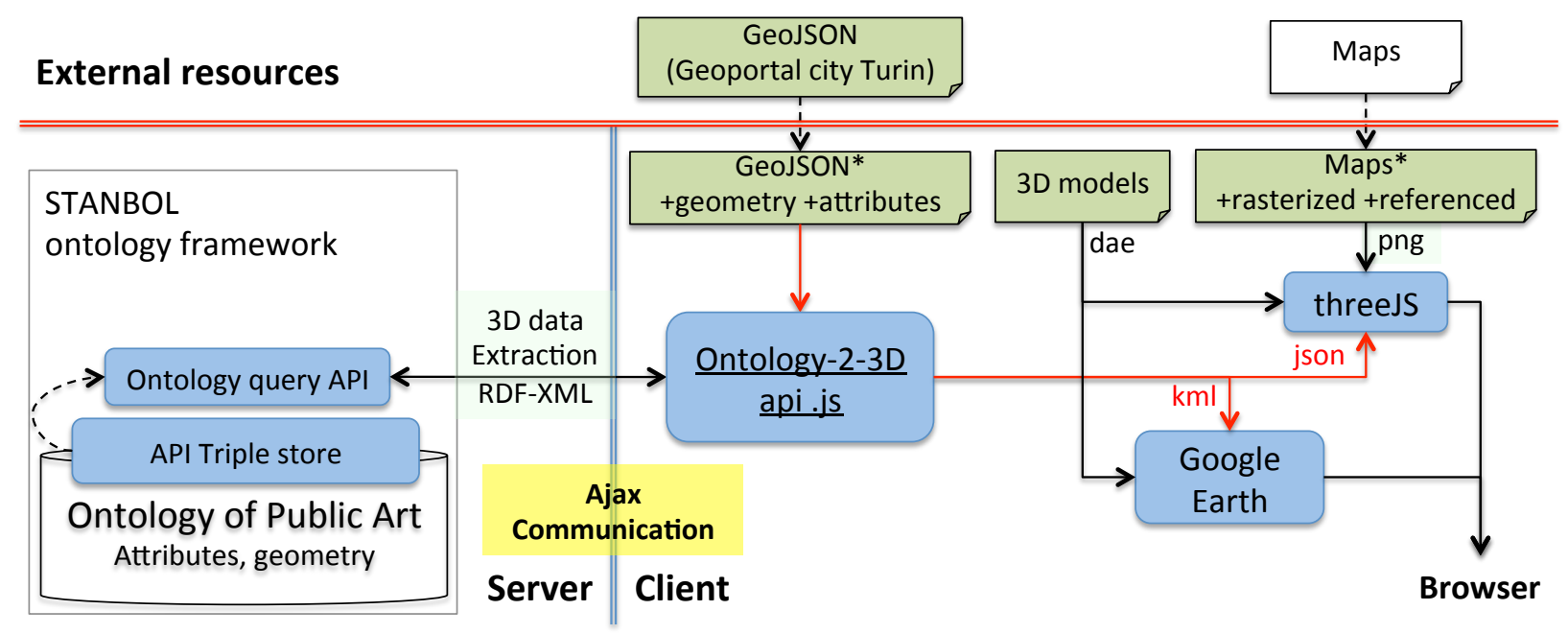

Fig. 5. Prototype architecture of visualization.

\section{THE VISUALIZATION SYSTEM}

The visualization component is embedded in a standard semantic architecture that encompasses three main modules (cf. [13]):

- the Ontology Server (implemented in the Stanbol framework $^{2}$ ) maintains the ontology "where the artworks are described" and provides the reasoning services; also, it provides a SPARQL endpoint for querying the ontology;

- the Web Service, written in Java, implements the API that client side applications exploit to query the Ontology Server. Depending on the project, the RDF triples extracted from the ontology are serialized as Json or XML data;

- $\quad$ the Visualization module supports the interaction with the user through 2D/3D navigation (or, else, standard hypertext including maps, timelines, etc.), as standalone application or embedded in a browser.

Within the architecture sketched above, the visualization module, then, relies on the following elements (Figure 5): 1) the ontology server makes available all the information needed for documenting and visualizing the artworks, including the including the 3D models, and is queried through specific commands in the API; 2) the visual renderer, developed in two versions: one, open-source, based on three.js library (where spatial data are encoded in a json data file); the other, proprietary, based on GoogleEarth (which requires a $\mathrm{kmz}$ file and does not need any maps); 3) the visual data, that is, the $3 \mathrm{D}$ models associated with items and artwork in the visual scene, the maps downloaded from some repository and employed through the Leaflet libraries, and the GeoJSON data extracted from the Geoportal of the city and augmented with information on geometry and other attributes. The user interface dynamically arranges a set of visual elements which embed the items, staging and setting them based on their properties, as represented in the ontology server, and offers

\footnotetext{
${ }^{2}$ https://stanbol.apache.org
}

the users a number of controls, as defined by the visualization framework.

The visualization framework consists of a set of offline components, that can be grouped into two main types: the visual components and the control interface. The visual components are the elements whose appearance depends upon the narrative metaphor, which drives the visual experience (e.g., the "city map" in the Invisibilia project). The visual components include: an environment, that provides the narrative context of the visualization where the visual objects are located; a set of visual objects, possibly with behaviors triggered by the user interaction; a scene layout, i.e., where objects are located in the environment. The control interface consists of the mapping between the artworks and the visual objects, actually the mapping of the artwork properties onto the visual features of the objects (color, size, shape, etc.), and of the relations among the artworks and the environment elements into spatial relations.

The design of the visual objects and of the scene layout for the Public Art, which are the result of the visualization module,

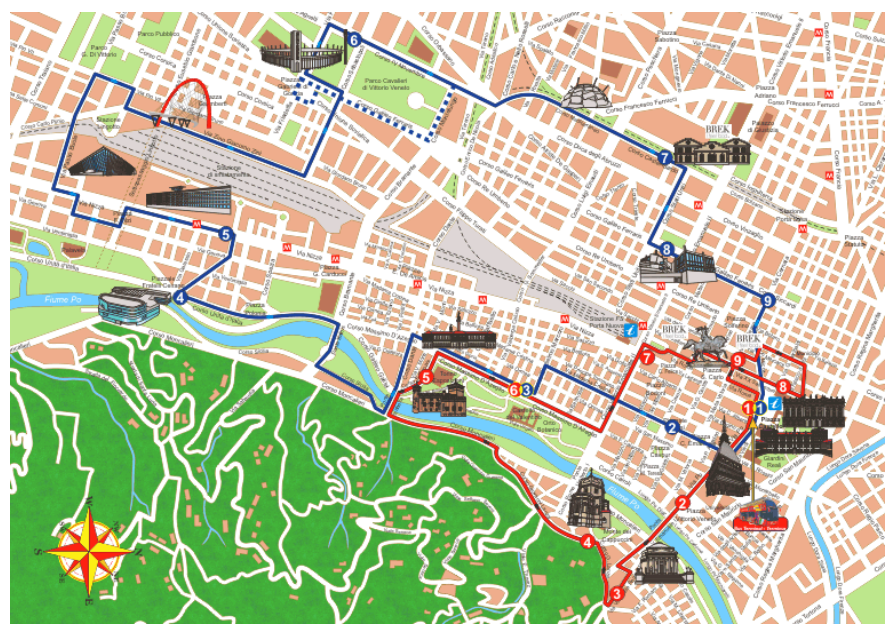

Fig. 6. Sightseeing map of the city of Turin. 


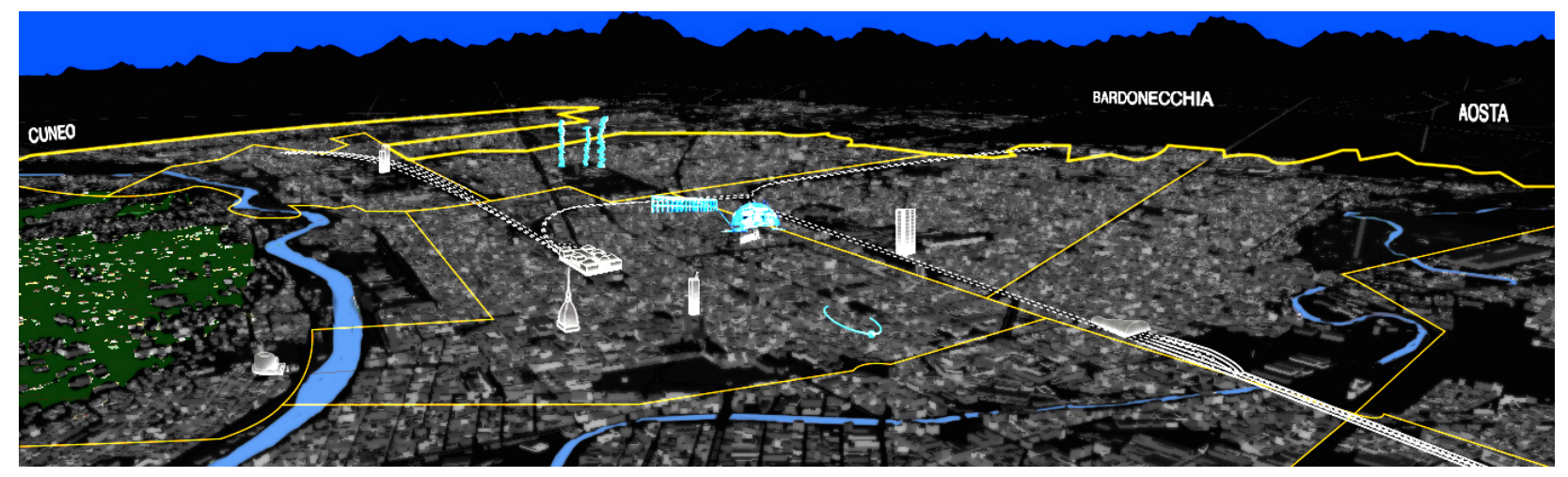

Fig. 7. Top level view. Design in Blender, visualized in three.js.

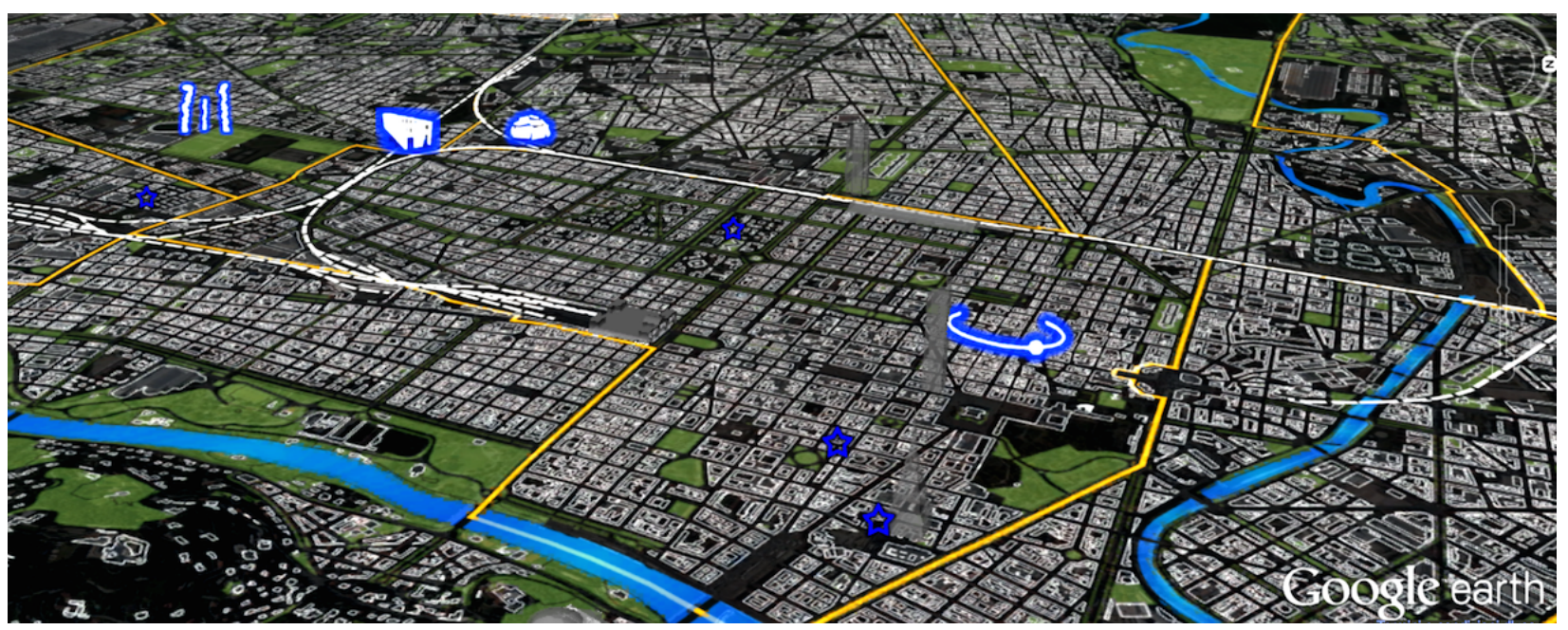

Fig. 8. Top level view. Design in SketchUp, visualized in Google Earth.

is an awkward phase. The design must reconcile the task of properly called visualization with the communication issues related with the documentation of the artworks. As described in the previous section, the tripartite ontology of Public Art has the goal of representing the documentation of the artworks, their urban context (functions, fruition, etc.) and the geospatial data about them: consequently, the same commitment to integration inspires the design of the user interface.

The public artworks come with a marked visual stance, which makes a visualization of the artwork documentation more challenging. We can sum up the following premises about the visualization: it cannot be a replacement for the original artwork, though exhibited in virtual terms; it has to balance between the goals of the visualization and peculiarities of the artwork; it must adhere to the model (of the features) of the artwork; the authorial intervention should be limited to the documentation section. The visual components that represent the characteristics of the artworks are derived from the existing documentation and are augmented with iconic elements appropriately designed by the visualization artist. These elements must be in overt contrast with the original elements, though creating an environment that can visually host the original elements; so, the idea is to acquire the artwork components and to integrate them in a single visual framework. Given the considerations above, we provide three visualization levels:

- the synoptic view, with the spatial layout of a number of artworks at the city level;

- $\quad$ an artwork view, for the exploration of an individual artwork;

- the documentation view, with the materials connected to the work-expression-manifestation tripartition, organized as a repository.

The synoptic view provides a top-view cartographic representation on a city map of the public artworks, with their relative positions; the public artworks are represented by visual items that show the shape of the artwork; these items can be original (if they exist in the documentation) or introduced ex-novo. The inspiration for this solution originates in the tourist city maps, that combine topological and topographical elements (see Figure $6^{3}$ ): in our case, we decided to employ Geoportal data from the Geographical web portal of the Municipality of Turin. To these we applied a graphical transformation, without any alteration on the geometry, in order to recall sightseeing touristic maps, which have a longstanding tradition in cartography and are effective in guiding tourists in picking the preferred sites in a city. Also, we augment such

${ }^{3}$ http://www.torino.city-sightseeing.it/eng/percorsi1.htm 


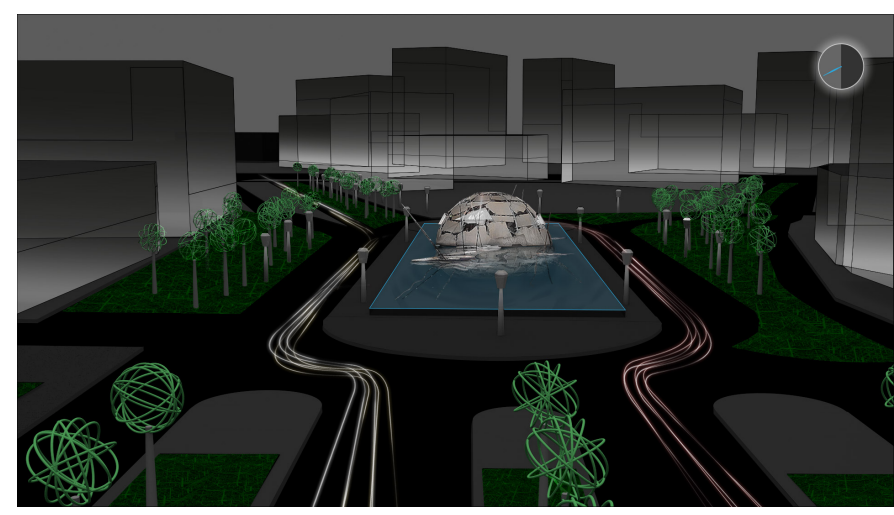

Fig. 9. Invisibilia artwork level view: design of Merz's Fountain in Blender.

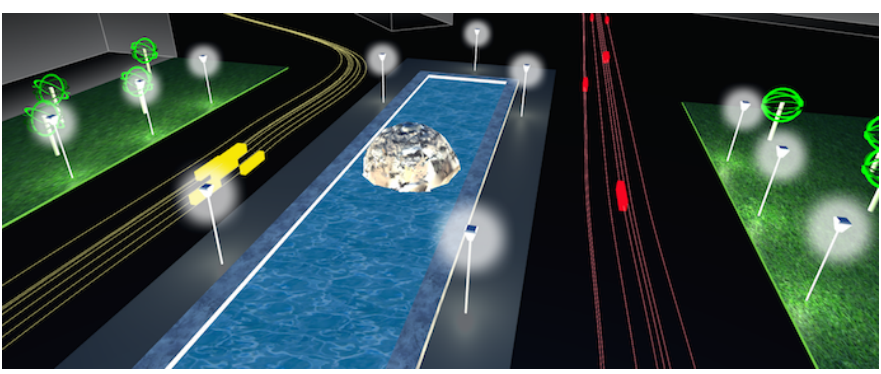

Fig. 10. Invisibilia artwork level view: implementation of Merz's Fountain in three.js library.

a visualization with a 3D perspective, augmented with data visualization techniques, in order to be coherent with the subsequent level and to make other invisible elements perceivable from the same map. For the synoptic view (see Figure 7), we insert topographic elements, such as the rivers (in blue), the major access as well as inner roads (in yellow and white), major reference buildings (in light grey), to provide orientation cues, and topological elements, one per public artwork with a light blue glow to provide a sense of presence against a dark background. In Figure 8, we see the implementation of the same scene in Google Earth; another implementation has been carried out in three.js library with maps from public repository imported through the Leaflet.js library (actually, in the current implementation, personalized maps are rasterized to cover a plan with an exact match of the references, given the relocation from the central point through a simple javascript function).

It is possible to go from the synoptic, top level view to the individual artwork view by clicking on the visual object that represents some artwork. The individual artwork view is a perspective view that is reached through a 3D-simulated camera motion: this view remains in the graphic style described above (monument in glow with a detail of the map), with superimposed active icons for accessing the visualization of the ontology features, namely work, expression, manifestation. In Figure 9, we can see the visualization conceived for Merz's Fountain, while in Figure 10 we can see the same design implemented in threejs library: the artwork is surrounded by roads, green areas, and tall buildings, and the latter elements are stylized icons, that repeat themselves over the several artworks (cf. Figure 11).

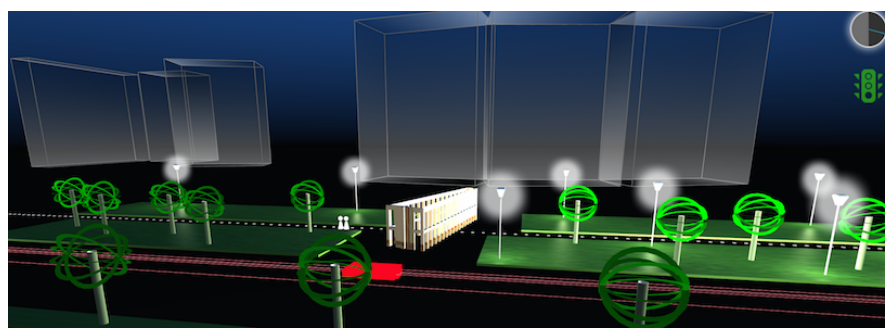

Fig. 11. Invisibilia artwork level view: implementation of Kirkeby's "Opera per Torino" in three.js library.

The object representing the artwork objects are 3D models with photographic texturing, obtained by means of automatic photogrammetric techniques. The input data are sets of digital images, acquired through a compact digital camera, which have been processed through the software Photoscan (see Figure 12). This approach, which can be carried out by a non $3 \mathrm{D}$ professional, such as the architects that work in the city hall office for Public Art, makes it sustainable from the operational point of view; the visual appearance makes the object different from the iconic solutions for the other elements.

The details of the documentation of the artwork can be accessed from the artwork level. The documentation usually includes several documents of various type, such as video clips, text documents, pictures, etc. These documents are related to the artwork concept, which illustrates the ideas of the artist, the production stages, which illustrate the various phases of the realization of the artwork, and the access plan, which, combined with the positioning provided by the top view and artwork level view, provides visitors with an idea of how to access the artwork (by foot, by car, etc.). The visualization interface displays the categories of document described above by following the tri-partition into work, expression, and manifestation that characterizes the FRBR model encoded in the CIDOC-CRM ontology. The Work material concerns the documentation about the artistic concept, i.e. the values the artwork conveys, expressed in terms of abstractions, sketches, related documents, including video inter-views with the artists (Figure 4, left); the Expression material concerns the production process, i.e. how the realization of the artwork unrolled over time, what materials were employed, what is the dynamics of the artwork); the Manifestation concerns the placement of the artwork in the physical site, with a characterization of the surroundings, audience access to the artwork, realization of the artwork. Each section is accessible through a dashboard that provides a view on all the documentation materials.

\section{CONCLUSION}

In this paper, we have described a knowledge-based framework for the documentation and communication of Public Art via a virtual reality environment. The knowledge about public art is encoded in a computational ontology consisting of three components: the documentation section, including the CIDOC CRM and FRBR models, the spatial component, which includes the visual elements of the scene that contains the artwork, the geographic section, with the coordinates of all the visual items. The visualization process has taken into account the placement of the artwork and the typology of the surroundings (park, buildings, and the height of buildings, green 


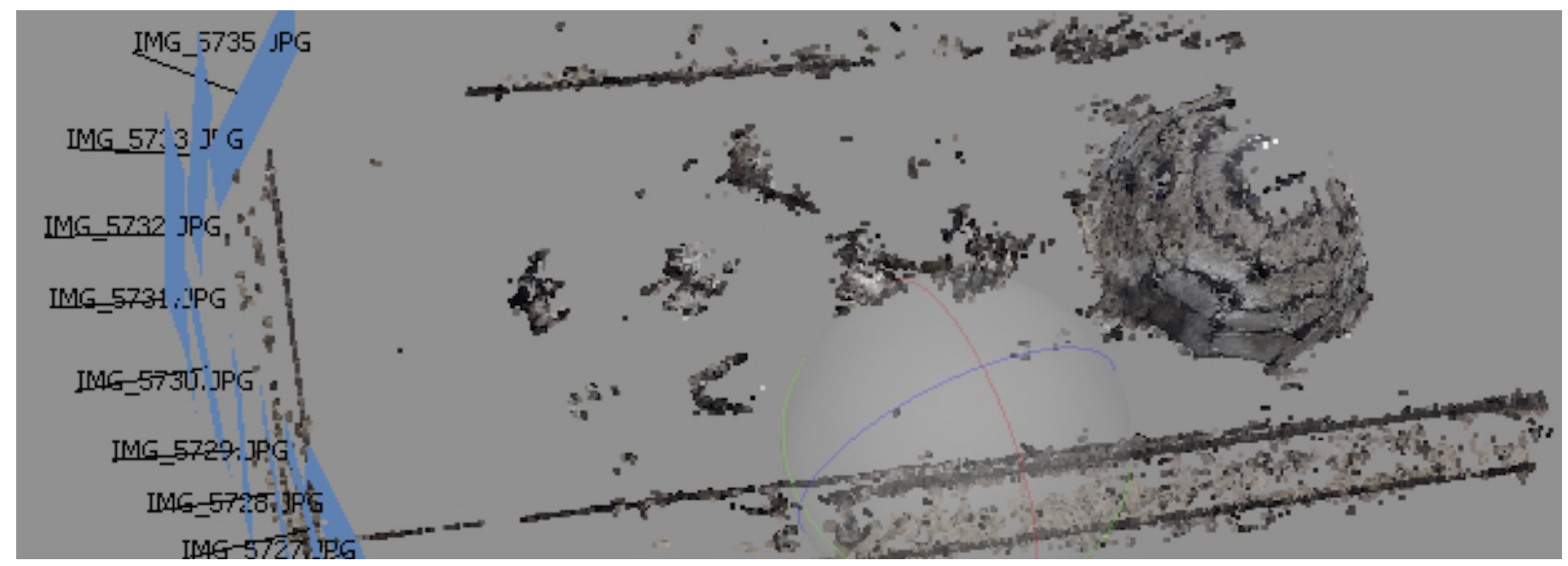

Fig. 12. Composition of Merz's Fountain scans in Photoscan.

areas), what are the methods and the ways for accessing and experiencing the artwork, sketches and video material about the conception and the realization of the concrete artwork. Public art also requires the exposition of the relationship of the artwork with respect to other public artworks in the same city. The visualization framework is arranged in three levels: a synoptic view, with the spatial layout of all the artworks at the city level; an artwork view, with the model of the artwork and standardized icons for the context model; the documentation view, with a dashboard of the documents about the artworks. We are going to test the visualization framework with professionals and users for the populating pipeline and the efficiency of the web site, respectively. It will also be interesting to address aspects related to system immersivity and alternative interaction methods, that provide access to further information about the public artworks.

\section{ACKNOWLEDGMENT}

Part of this research was funded through the Invisibilia Project (2012-2014), supported by Compagnia di San Paolo, Progetti di ricerca di ateneo (2011), DR n. 247 28.01.2011. We thank Giulio Lughi, coordinator of the project. We thank Alessandro Olivero and Nadia Guardini for their implementation of the virtual environment. We also thank Massimo Morelli, Adriana Ramundo e Valentino Chiarla, of Pensativa s.r.l., for their collaboration and support in the implementation process.

\section{REFERENCES}

[1] M. Miles, Art, space and the city: public art and urban futures. Psychology Press, 1997.
[2] V. Lombardo, A. Valle, F. Nunnari, F. Giordana, and A. Arghinenti, "Archeology of multimedia," in Proceedings of the Association for Computing Machinery Multimedia Conference 2006 (ACM-MM 2006). Santa Barbara, CA, USA: Association for Computing Machinery, October 2006, pp. 269-278.

[3] L. M. Chan, Cataloging and classification: an introduction. Scarecrow Press, 2007.

[4] M. Doerr, "The cidoc conceptual reference module: an ontological approach to semantic interoperability of metadata," AI magazine, vol. 24, no. 3 , p. $75,2003$.

[5] T. Berners-Lee, J. Hendler, O. Lassila et al., "The semantic web," Scientific american, vol. 284, no. 5, pp. 28-37, 2001.

[6] M. Doerr, "Ontologies for cultural heritage," in Handbook on Ontologies. Springer, 2009, pp. 463-486.

[7] E. Hyvönen, "Semantic portals for cultural heritage," in Handbook on ontologies. Springer, 2009, pp. 757-778.

[8] M. Theodoridou, Y. Tzitzikas, M. Doerr, Y. Marketakis, and V. Melessanakis, "Modeling and querying provenance by extending cidoc crm," Distributed and Parallel Databases, vol. 27, no. 2, pp. 169-210, 2010.

[9] K. Ng, T. V. Pham, B. Ong, and A. Mikroyannidis, "Preservation of interactive multimedia performances," International Journal of Metadata, Semantics and Ontologies, vol. 3, no. 3, pp. 183-196, 2008.

[10] C. Doty, "The difficulty of an ontology of live performance," InterActions: UCLA Journal of Education and Information Studies, vol. 9, no. $1,2013$.

[11] E. T. O'Neill, "Frbr: Functional requirements for bibliographic records," Library resources \& technical services, vol. 46, no. 4, pp. 150-159, 2002.

[12] M. Doerr and P. LeBoeuf, "Modelling intellectual processes: the frbrcrm harmonization," in Digital libraries: Research and development. Springer, 2007, pp. 114-123.

[13] R. Damiano, A. Lieto, and V. Lombardo, "Ontology-based visualisation of cultural heritage," in Eighth International Conference on Complex, Intelligent and Software Intensive Systems. IEEE, 2014, pp. 558-563. 